\title{
A THEOREM ON CLUSTER SETS OF AN ANALYTIC MAPPING INTO A RIEMANN SURFACE
}

\section{MAKOTO OHTSUKA}

1. Introduction. The author [5] proved in 1952 that if an analytic mapping $f$ of a punctured disk $0<|z|<r$ into a Riemann surface $R$ has $z=0$ as an essential singularity in a certain sense, then the set of values in $R$ taken by $f$ in any neighborhood of $z=0$ is conformally equivalent to a sphere possibly less two points or to a torus. Heins [1] and Marden, Richards and Rodin [2] gave different proofs and the latter applied the above result to the study of analytic self-mappings of Riemann surfaces.

In the present paper we shall treat the case where the singularity is not isolated. Our result gives a generalization of a theorem of Noshiro (see Theorem 6 at p. 26 of [4]). To explain his result, let $f$ be a meromorphic function defined in a domain $D$, and $K$ a compact set of logarithmic capacity zero on one component of the boundary $\partial D$. Let $z_{0}$ be a point of $K$ not isolated on $(\partial D-K) \cup\left\{z_{0}\right\}$. Then it can be shown that the difference $\Omega$ between the cluster set at $z_{0}$ and the boundary cluster set defined at $z_{0}$ along $\partial D-K$ is an open set. Noshiro proved that $f$ takes every value, with two possible exceptions, of each component of $\Omega$ in any neighborhood of $z_{0}$.

2. Preliminaries. Let $R$ be a finite Riemann surface, and $\varrho_{z}|d z|$ be a conformal metric on $R$ with strictly positive coefficient $\varrho_{z}$. Let $S$ be a covering surface of $R$. One can regard $\varrho_{z}|d z|$ as a conformal metric on $S$. The coefficient may vanish on $S$; actually it does at each branch point of $S$. We set $L(c)=\int_{c} \varrho_{z}|d z|$ for a smooth arc $c$ on $R$ and $I(E)=\iint_{E} \varrho_{z}^{2} d x d y$ for a measurable set $E$ on $R$. We shall use the same notation for such quantities on $S$ too.

Let $S$ be a simply connected finite Riemann surface in particular. Ahlfors' main theorem asserts that there exists a constant $h$ depending only on $R$ such that

$$
0 \geqq e_{0} M(S)-h L(\partial S),
$$


where $e_{0}$ is the characteristic of $R, M(S)=I(S) / I(R)$ is the mean sheet number of $S$ and $\partial S$ is the boundary of $S$ relative to $R$.

Let us call a simply connected domain on $R$ with analytic boundary an open disk. Take open disks $\Delta_{1}, \ldots, \Delta_{q}$ on $R$ whose closures are mutually disjoint and contained in the interior of $R$. Denote the projection of $S$ into $R$ by $f$. A component of $f^{-1}\left(\Delta_{j}\right)$ is called an island lying above $\Delta_{j}$ if its boundary consists of inner points of $S$. Let $\left\{D_{i}\right\}$ be the islands lying above $\Delta_{1}, \ldots, \Delta_{q}$. By the aid of (1) we derive

$$
-\sum e\left(D_{i}\right) \geqq\left(e_{0}+q\right) M(S)-h L(\partial S),
$$

where $e\left(D_{i}\right)$ is the characteristic of $D_{i}$; if there is no island, then the left hand side is set to be zero. See (60) of [6] for our (2).

Let $S$ now be a simply connected bordered covering surface of $R$. We call an increasing approximation $\left\{S_{n}\right\}$ of $S$ a regular exhaustion of $S$ if each $S_{n}$ consists of finitely many finite surfaces and

$$
\frac{L\left(\partial S_{n}\right)}{M\left(S_{n}\right)} \rightarrow 0 \quad \text { as } n \rightarrow \infty,
$$

where $\partial S_{n}$ is the boundary of $S_{n}$ relative to $R$. We note that our definition is general in the sense that $S_{n}$ may not be connected. When there exists a regular exhaustion of $S$, we say that $S$ is regularly exhaustible.

We have

L e m m a . Let $S$ be a regularly exhaustible simply connected bordered Riemann surface which is a covering surface of a finite Riemann surface $R$ of characteristic $e_{0}$. Then $e_{0} \leqq 0$ and $S$ covers all inner points of $R$ except at most $-e_{0}$ points.

Proof. Let $\left\{S_{n}\right\}$ be a regular exhaustion. From (1) it follows that $e_{0} \leqq 0$. Next, assume that $S$ does not cover $q=1-e_{0}$ inner points of $R$, and take disks $\Delta_{1}, \ldots, \Delta_{q}$ around them as above. Then there is no island above $\Delta_{1}, \ldots, \Delta_{q}$. By $(2)$ we have $0 \geqq M\left(S_{n}\right)-h L\left(\partial S_{n}\right)$ and meet a contradiction.

3. Main theorem. Let $G$ be an open set in the $z$-plane, $z_{0} \in \partial G$ and $K$ a compact subset of $\partial G$ containing $z_{0}$. Let $f$ be a mapping of $G$ into a Riemann surface $R$. We define the cluster set $f\left(z_{0} ; G\right)$ at $z_{0}$ to be

$$
\bigcap_{U \in \mathscr{U}\left(z_{0}\right)} \overline{f(U \cap G)}
$$

where $\mathscr{U}\left(z_{0}\right)$ is the system of neighborhoods of $z_{0}$ and $\overline{f(U \cap G)}$ denotes the closure of $f(U \cap G)$, and define a boundary cluster set $f\left(z_{0} ; \partial G-K\right)$ by

$$
\bigcap_{U \in \mathscr{U}\left(z_{0}\right)} \overline{\bigcup_{z \in U}} f(z ; G) .
$$


We shall prove

Th e o r e m. Let $f$ be an analytic mapping of an open set $G$ in the z-plane into a Riemann surface $R, z_{0} \in \partial G$ and $K$ a compact set of logarithmic capacity zero which contains $z_{0}$ and which is contained in one component of $\partial G$. If $f\left(z_{0} ; G\right)$ contains more than one point, then $f\left(z_{0} ; G\right)$ $f\left(z_{0} ; \partial G-K\right)$ is an open set and the genus of every component $D$ of $f\left(z_{0} ; G\right)$ $f\left(z_{0} ; \partial G-K\right)$ is at most one. If the genus is 0 ( 1 resp.), then every point of $D$ is taken by $f$ in any neighborhood of $z_{0}$ except for at most two (with no exception resp.).

Proof. Suppose $f\left(z_{0} ; G\right)-f\left(z_{0} ; \partial G-K\right)$ is not open. Then the boundary $\partial f\left(z_{0} ; G\right)$ is not contained in $f\left(z_{0} ; \partial G-K\right)$. Let $P_{0}$ be a point of $\partial f\left(z_{0} ; G\right)-f\left(z_{0} ; \partial G-K\right)$, and $N$ be a closed neighborhood of $P_{0}$ which does not include the whole $f\left(z_{0} ; G\right)$ and which is disjoint from $f\left(z_{0} ; \partial G-K\right)$. Let $w$ be a local parameter such that $N$ contains the local disk which corresponds to $|w| \leqq 1$ and $P_{0}$ corresponds to $w=0$. Denote the composed mapping $w(f(z))$ by $g(z)$, and let $\Omega$ be the inverse image of $|w|<1$ in $G$. Since $P_{0} \in f\left(z_{0} ; G\right)$, there exists a sequence $\left\{z_{n}\right\}$ in $G$ tending to $z_{0}$ such that $f\left(z_{n}\right) \rightarrow P_{0}$. Hence $z_{0}$ is a boundary point of $\Omega$. Suppose $z_{0}$ is isolated on $(\partial \Omega-K) \cup\left\{z_{0}\right\}$. Then $\Omega \cap U=U-K=$ $G \cap U$ for some neighborhood $U$ of $z_{0}$, and hence $f\left(z_{0} ; G\right) \subset N$. This is against our choice of $N$. Thus $z_{0}$ is not isolated on $(\partial \Omega-K) \cup\left\{z_{0}\right\}$. We see that $g\left(z_{0} ; \partial \Omega-K\right)$ is contained in $|w|=1$ and that $w=0$ belongs to $\partial g\left(z_{0} ; \Omega\right)$. Thus $\partial g\left(z_{0} ; \Omega\right) \notin g\left(z_{0} ; \partial G-K\right)$. However, if we use Theorem 4 at p. 17 of [4] in the theory of cluster sets for functions, then we conclude that $\partial g\left(z_{0} ; \Omega\right) \subset g\left(z_{0} ; \partial \Omega-K\right)$. This contradiction shows that $f\left(z_{0} ; G\right)-f\left(z_{0} ; \partial G-K\right)$ is open.

We shall prove next that, in case the genus of a component $D$ of $f\left(z_{0} ; G\right)-f\left(z_{0} ; \partial G-K\right)$ is zero, $f$ takes on every value of $D$ except for at most two in any neighborhood of $z_{0}$. The proof is exactly the same as for Noshiro's theorem referred to in the introduction. For the sake of completeness, however, we shall prove it. Suppose $P_{1}, P_{2}, P_{3} \in D$ are not taken by $f$ on $G \cap\left\{\left|z-z_{0}\right| \leqq r\right\}$. Draw an analytic simple closed curve $c$ which passes through $P_{3}$, whose interior $\Delta$ contains $P_{1}$ and $P_{2}$ and above which lies no branch point of $G$ as a covering surface of $R$. We take $c$ in $D$ so that $\Delta$ is included in $D$. Since $D \subset f\left(z_{0} ; G\right)$, there is a sequence $\left\{z_{k}\right\}$ tending to $z_{0}$ whose image $\left\{f\left(z_{k}\right)\right\}$ is contained in $\Delta$ and tends to $P_{1}$. We may assume that no $z_{k}$ is a branch point. Let $\left(f\left(z_{k}\right) P_{1}\right)^{\sim}$ be a curve in $D$ which passes through no image of any branch point and converges to $P_{1}$ as $k \rightarrow \infty$, and let $\gamma_{k}$ be the inverse image of $\left(f\left(z_{k}\right) P_{1}\right)^{\sim}$ starting from $z_{k}$. If there are infinitely many $\gamma_{k}$ which intersect $\left|z-z_{0}\right|=r$, these $\gamma_{k}$ cluster to a continuum $F$ connecting $z_{0}$ and $\left|z-z_{0}\right|=r$. Since $f$ is not constant, $F$ has no point in $G$. Accordingly 
$F \subset \partial G$. We see that every $U \in \mathscr{U}\left(z_{0}\right)$ contains some point $z$ of $F-K$ and $f(z ; G)$ contains $P_{1}$ at such $z$. Accordingly $f\left(z_{0} ; \partial G-K\right)$ contains $P_{1}$. This is impossible. It follows that $\gamma_{k}$ must terminate at a point of $K \cap\left\{\left|z-z_{0}\right|<r\right\}$ if $k$ is large. Let $S$ be a component of

$$
f^{-1}(\bar{\Delta}) \cap\left\{\left|z-z_{0}\right| \leqq r\right\}
$$

which contains such a $\gamma_{k}$. Since $f$ does not assume $P_{3}$ on $G \cap\left\{\left|z-z_{0}\right| \leqq\right.$ $r\}, S$ is simply connected.

Let a conformal metric $\varrho_{w}|d w|$ be given on $\Delta$. Let us see that $S$ is regularly exhaustible as a covering surface of $\Delta$. It is well known that there exists a logarithmic potential $U(z)$ of a unit measure supported by $K$ such that $U=\infty$ on $K$. Let $V$ be a conjugate of $U$ and let $\zeta=F(z)$ be a single-valued branch in $S$ of $\exp (U(z)+i V(z))$. If $\lambda_{0}$ is large, then the level set $\left\{z ; e^{U(z)}=\lambda\right\}$ intersects $\gamma_{k}$ for every $\lambda \geqq \lambda_{0}$. Take $\zeta$ as a local parameter at every point $z$ of $S$ at which $F^{\prime}(z) \neq 0$, i.e., grad $U \neq 0$. Denote the part of $S$ on which $e^{U}<\lambda$ by $S_{\lambda}$, and the level set $\left\{z \in S ;|F(z)|=e^{U(z)}=\lambda\right\}$ by $\Theta_{\lambda}$. Denoting by $(\lambda, \vartheta)$ the polar coordinates in the $\zeta$-plane, we have

$$
I\left(S_{\lambda}\right)-I\left(S_{\lambda_{0}}\right)=\int_{\lambda_{0}}^{\lambda} \int_{\Theta_{\lambda}} \varrho_{\zeta}^{2} \lambda d \vartheta d \lambda
$$

where $\varrho_{\zeta}|d \zeta|$ is the conformal metric on $S$ expressed in terms of $\zeta$ and equals $\varrho_{w}|d w|$ at every point $z$ at which $F^{\prime}(z) \neq 0$ and which is not a branch point of $f$. Set

$$
L(\lambda)=\int_{\Theta_{\lambda}} \varrho_{\zeta} \lambda d \vartheta
$$

Denote by $\alpha>0$ the distance between $c$ and $\left(f\left(z_{k}\right) P_{1}\right)^{\sim}$, measured with respect to $\varrho_{w}|d w|$. Then $L(\lambda) \geqq 2 \alpha$ for $\lambda \geqq \lambda_{0}$. Applying Schwarz's inequality we derive

$$
(L(\lambda))^{2} \leqq \int_{\Theta_{\lambda}} \lambda d \vartheta \int_{\Theta_{\lambda}} \varrho_{\zeta}^{2} \lambda d \vartheta=\lambda \vartheta(\lambda) \int_{\Theta_{\lambda}} \varrho_{\zeta}^{2} \lambda d \vartheta
$$

where $\vartheta(\lambda)=\int_{\Theta_{\lambda}} d \vartheta$, and

$$
\frac{(L(\lambda))^{2}}{\lambda \vartheta(\lambda)} \leqq \int_{\Theta_{\lambda}} \varrho_{\zeta}^{2} \lambda d \vartheta=\frac{d I\left(S_{\lambda}\right)}{d \lambda}
$$

Using the relation 


$$
\vartheta(\lambda)=\cdot \int_{\Theta_{\lambda}} d V \leqq \int_{U=\log \lambda} \frac{\partial U}{\partial n} d s=2 \pi,
$$

we have

$$
\frac{2 \alpha^{2}}{\pi} \int_{\lambda_{0}} \frac{d \lambda}{\lambda} \leqq \int_{\lambda_{0}}^{\lambda} d I\left(S_{\lambda}\right)=I\left(S_{\lambda}\right)-I\left(S_{\lambda_{0}}\right) .
$$

It follows that $I\left(S_{\lambda}\right) \rightarrow \infty$ as $\lambda \rightarrow \infty$. If there existed $\beta>0$ and $\lambda_{1}>\lambda_{0}$ such that $I\left(S_{\lambda}\right) \leqq \beta L(\lambda)$ for all $\lambda \geqq \lambda_{1}$, then

$$
\frac{1}{2 \pi} \int_{\lambda_{1}}^{\lambda} \frac{d \lambda}{\lambda} \leqq \beta^{2} \int_{\lambda_{1}}^{\lambda} \frac{d I\left(S_{\lambda}\right)}{\left(I\left(S_{\lambda}\right)\right)^{2}} \leqq \frac{\beta^{2}}{I\left(S_{\lambda_{1}}\right)}<\infty .
$$

This is absurd. Therefore there exists $\left\{\lambda_{n}\right\}$ tending to $\infty$ such that

$$
\frac{L\left(\partial S_{\lambda_{n}}\right)}{I\left(S_{\lambda_{n}}\right)}=\frac{L\left(\lambda_{n}\right)+L\left(\partial S \cap\left\{\left|z-z_{0}\right|=r\right\}\right)}{I\left(S_{\lambda_{n}}\right)} \rightarrow 0 \text { as } n \rightarrow \infty .
$$

Thus $S$ is a regularly exhaustible simply connected bordered covering surface of $\bar{\Delta}$. Our lemma implies that $S$ covers all points of $\Delta$ except at most one point. This is not true. Consequently, every component of $f\left(z_{0} ; G\right)-f\left(z_{0} ; \partial G-K\right)$ of genus zero is covered by the image of any neighborhood of $z_{0}$ except for at most two points.

Secondly, let $D$ be a component of $f\left(z_{0} ; G\right)-f\left(z_{0} ; \partial G-K\right)$ of genus at least one, and assume that $P_{0} \in D$ is not taken by $f$ in $G \cap\left\{\left|z-z_{0}\right| \leqq r\right\}$. Take a subdomain $\Delta$ of genus one of $D$ bounded by an analytic simple closed curve $c$ in $D$ which passes through $P_{0}$ and above which lies no branch point of $G$. If there is $P_{1} \in \Delta$ which is not taken by $f$ in a neighborhood of $z_{0}$, then we observe that there is a curve $\gamma$ in any neighborhood of $z_{0}$ which terminates at a point of $K$ and along which $f$ tends to $P_{1}$. Let $S$ be a component of $f^{-1}(\bar{\Delta}) \cap\left\{\left|z-z_{0}\right| \leqq r\right\}$ containing $\gamma$. It is simply connected. We can show as above that it is a regularly exhaustible bordered covering surface of $\bar{\Delta}$. Since the characteristic of $\Delta$ is one, our lemma gives a contradiction. Hence all points of $\Delta$ are taken in any neighborhood of $z_{0}$. Since $\Delta$ contains a topological handle, there are analytic simple closed curves $c_{1}$ and $c_{2}$ in $\Delta$ such that they intersect mutually only at a point $P_{2}$ and no branch point of $G$ lies above $c_{1} \cup c_{2}$. Let $\left\{z_{k}\right\}$ be a sequence of points tending to $z_{0}$ such that $f\left(z_{k}\right)=P_{2}$ for each $k$. Consider the component $l_{k}$ of $f^{-1}\left(c_{1}\right)$ which passes through $z_{k}$. Suppose there is no $l_{k}$ which terminates at $K$ for large $k$. Then for some $k_{0} l_{k_{0}}$ must be a closed curve in $G \cap\left\{\left|z-z_{0}\right|<r\right\}$. Consider the component $l_{k_{0}}^{\prime}$ of $f^{-1}\left(c_{2}\right)$ which starts from $z_{k_{0}}$ and runs in the interior 
$D_{k_{0}}$ of $l_{k_{0}}$. Since the part of $D_{k_{0}}$ near $l_{k_{0}}$ corresponds to one shore of $c_{1}, l_{k_{0}}^{\prime}$ can not intersect $l_{k_{0}}$ again. Hence it must terminate at some point of $K$. It is now concluded that, in any neighborhood of $z_{0}$, there is a curve which terminates at a point of $K$ and whose image by $f$ is contained in $c_{1}$ or $c_{2}$. Let $S$ be a component of $f^{-1}(\bar{\Delta}) \cap\left\{\left|z-z_{0}\right| \leqq r\right\}$ containing such a curve. It is a simply connected regularly exhaustible bordered covering surface of $\bar{\Delta}$. This is again impossible. Thus every point of $D$ is taken in any neighborhood of $z_{0}$.

Finally, suppose the genus of a component $D$ is at least two. Take a subdomain $\Delta$ of genus one of $D$ bounded by an analytic simple closed curve $c$ in $D$. Suppose there is a component $S_{0}$ of

$$
f^{-1}(\bar{\Delta}) \cap\left\{\left|z-z_{0}\right| \leqq r\right\}
$$

which is not simply connected. Then there exists a closed curve $\gamma^{\prime}$ on $\partial S_{0}$ corresponding to $c$. Take a non-branch point $z^{\prime} \in S_{0}$ close to $\gamma^{\prime}$ and let $P$ be its image. Since $D-\bar{\Delta}$ is not planar, it contains two analytic simple closed curves $c_{1}^{\prime}$ and $c_{2}^{\prime}$ which meet only at $P$ and above which no branch point of $G$ lies. The inverse image of $c_{1}^{\prime}$ passing through $z^{\prime}$ must be a closed curve. If we start from $z^{\prime}$ in one direction along the inverse image of $c_{2}^{\prime}$, we have no place to go after all. Consequently, every component of $f^{-1}(\bar{\Delta}) \cap\left\{\left|z-z_{0}\right| \leqq r\right\}$ is simply connected. The rest of the proof is the same as above. The proof of our theorem is now completed.

Remark 1. In our theorem the case when $f\left(z_{0} ; G\right)$ consists of a single point or is empty is not treated. Therefore it does not include author's result in [5]. See [5] in this aspect.

Remark 2. In [5] the author stated that he could not apply Ahlfors' theory of covering surfaces to prove Theorem 1. The present paper surmounts that difficulty.

Remark 3. If the condition that $K$ is contained in one component of $\partial G$ is removed in our theorem, then the conclusion is not true in general. Actually, Matsumoto [3] proved that, given any $K_{\sigma}$-set $E$ of logarithmic capacity zero in the $w$-plane, there exist a compact set $K$ of logarithmic capacity zero in the $z$-plane and a meromorphic function $w=f(z)$ defined outside $K$ such that every point of $K$ is a singularity for $f$ and $E$ is the set of exceptional values at each point of $K$.

In connexion with this remark we mention the following open question due to M. Suzuki:

Let $D$ be a domain in a plane and $K$ be a compact subset of $D$ of logarithmic capacity zero. Let $f$ be an analytic mapping of $D-K$ into a Riemann surface $R$ such that the cluster set of $f$ at a point of $K$ coincides with $R$. Then, is the genus of $R$ at most one? 


\title{
References
}

[1] HeIns, M.: On Fuchsoid groups that contain parabolic transformations. - Contributions to function theory. Tata Institute of Fundamental Research, Bombay, 1960, 203-210.

[2] MARDen, M., I. Richards and B. Rodin: Analytic self-mappings on Riemann surfaces. - J. Analyse Math. 18, 1967, 197-225.

[3] Matsumoto, K.: Exceptional values of meromorphic functions in a neighborhood of the set of singularities. - J. Sci. Hiroshima Univ. Ser. A 24, 1960, $143-153$.

[4] Noshiro, K.: Cluster sets. - Ergebnisse der Mathematik und ihrer Grenzgebiete (Neue Folge) 28. Springer-Verlag, Berlin - Göttingen - Heidelberg, 1960.

[5] OHTSUKA, M.: On the behavior of an analytic function about an isolated boundary point. - Nagoya Math. J. 4, 1952, 103-108.

[6] SarIo, L., and K. Noshiro: Value distribution theory. - The University Series in Higher Mathematics. D. Van Nostrand Company, Inc., Princeton, New Jersey, etc., 1966.

\author{
Hiroshima University \\ Faculty of Science \\ Department of Mathematics \\ Hiroshima \\ Japan 730
}

Received 2 September 1975 